\section{Mycobacterium neoaurum Causing Prosthetic Valve Endocarditis}

\author{
Anupam Kumar* \\ University of Connecticut Health Center, USA
}

\begin{abstract}
Mycobacterium neoaurum rarely causes bacteremia, and infection usually occurs in immunocompromised hosts. Prosthetic Valve Endocarditis (PVE) due to Non Tuberculous Mycobacteria (NTM) is rare; Mycobacterium neoaurum has never been implicated in this disease. We report a case of Mycobacterium neoaurum prosthetic valve endocarditis with favorable response to therapy.
\end{abstract}

\section{Case Report}

A 30 year old Caucasian male presented to the emergency department with shortness of breath and palpitations of 2 weeks duration associated with fever and chills. His past medical history was significant for multiple episodes of MRSA endocarditis in the setting of intravenous drug use, for which he had undergone bovine mitral valve replacement a year prior. He admitted to continued intravenous drug use despite recurrent endocarditis and valve replacement. His vital signs on admission were temperature of $101.4^{\circ} \mathrm{F}$, pulse rate of 106 per minute, blood pressure of $98 / 58 \mathrm{~mm} \mathrm{Hg}$ and respiratory rate of 20 per minute. A Grade 3/6 holosystolic murmur was heard best at the mitral area and radiated to the axilla. The lower extremities were warm to touch. Distal pulses were intact and symmetric. A detailed neurological exam was unremarkable. Admission laboratory tests showed a WBC count of $16.8 \mathrm{~K} / \mathrm{mm} 3$ with neutrophil predominance $(84 \%)$. Serum electrolytes, blood urea nitrogen, creatinine, creatine kinase (CK) and liver function tests were within the normal range. Chest $\mathrm{x}$-ray showed no abnormality, and electrocardiogram showed normal sinus rhythm with mild left atrial enlargement.

Blood cultures were drawn and the patient was started on empiric antibiotic therapy with vancomycin, ceftriaxone and rifampin for provisional diagnosis of prosthetic valve endocarditis. A transthoracic echocardiogram revealed extensive vegetations on the prosthetic mitral valve with mild obstruction to mitral inflow and a normal ejection fraction (Figure 1). The gram stain of the blood smear was initially reported as rods with both gram positive and gramnegative characteristics (Figure 2). However, the acid-fast stain turned positive and three blood culture bottles (aerobic) eventually grew Mycobacterium species (Figure 3). The organism was further identified as Mycobacterium neoaurum using the 16s ribosomal DNA sequencing method. The patient was treated with an anti-mycobacterial regimen based on susceptibility data: IV tobramycin, oral azithromycin

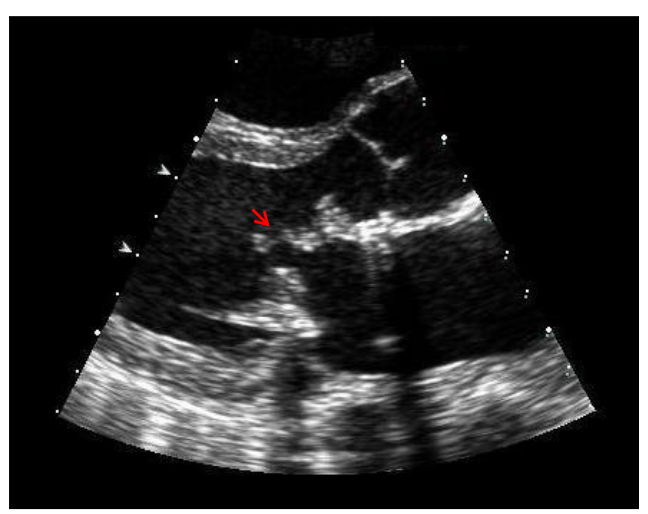

Figure 1: Echocardiogram showing vegetations on the prosthetic mitral valve with mild obstruction to mitral inflow.

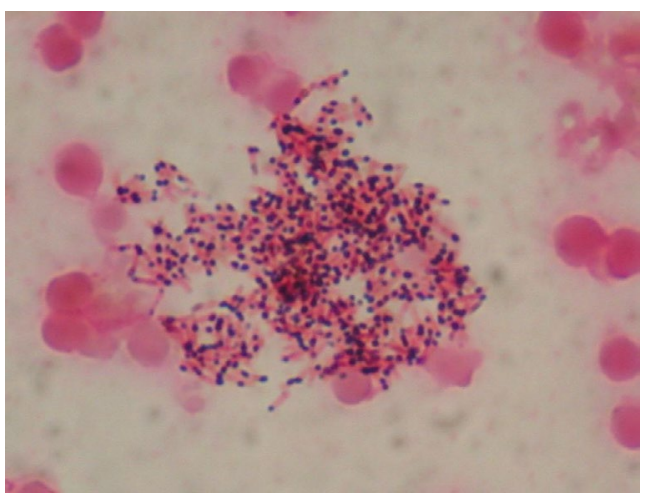

Figure 2: Gram stain showing organism with gram positive and gram negative characteristics.

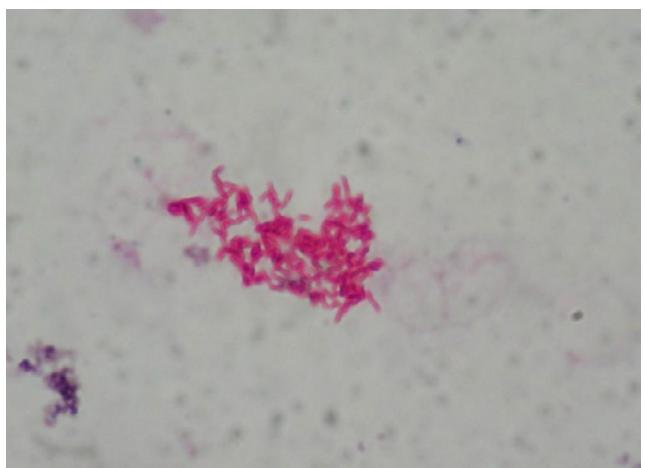

Figure 3: Ziehl Neelsen staining demonstrating acid fast organisms suggestive of mycobacteria species.

and oral moxifloxacin. On day ten of his hospital stay he developed severe abdominal pain. CT scan of the abdomen revealed a splenic infarct (Figure 4) as well as left renal infarct (Figure 5). MRI of brain revealed one centimeter brain abscess (Figure 6). Surgical intervention was discouraged given his ongoing drug use. The patient's condition

${ }^{*}$ Corresponding author: AnupamKumar, University of Connecticut Health Center, 263 Farmington Ave Farmington, 06030 CT, USA, E-mail: drkumar83@gmail.com

Received January 28, 2013; Accepted August 23, 2013; Published August 25, 2013

Citation: Anupam Kumar (2013) Mycobacterium neoaurum Causing Prosthetic Valve Endocarditis. J Pulmon Resp Med S14: 006. doi:10.4172/2161-105X.S14006

Copyright: () 2013 Anupam Kumar. This is an open-access article distributed under the terms of the Creative Commons Attribution License, which permits unrestricted use, distribution, and reproduction in any medium, provided the original author and source are credited. 


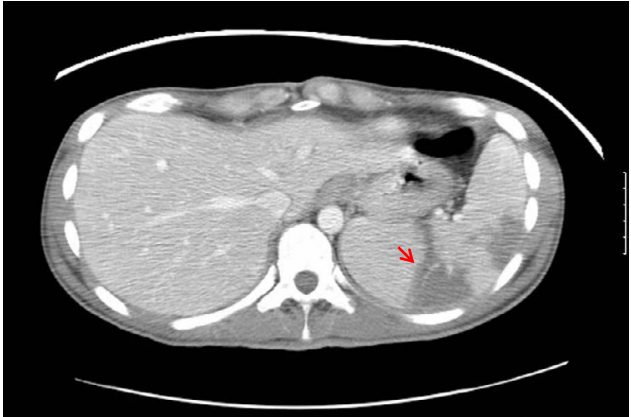

Figure 4: CT scan of abdomen showing infarct of the spleen

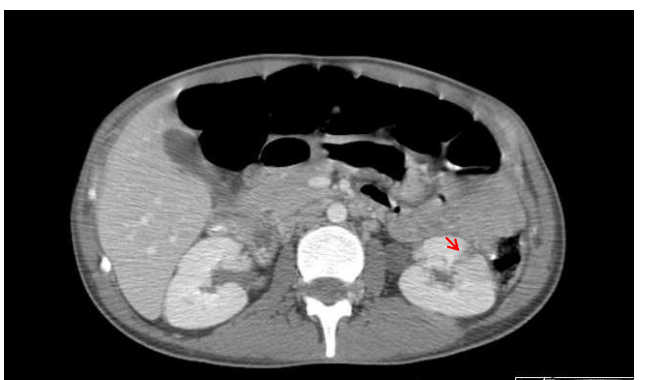

Figure 5: CT scan of the abdomen showing left renal infarct.

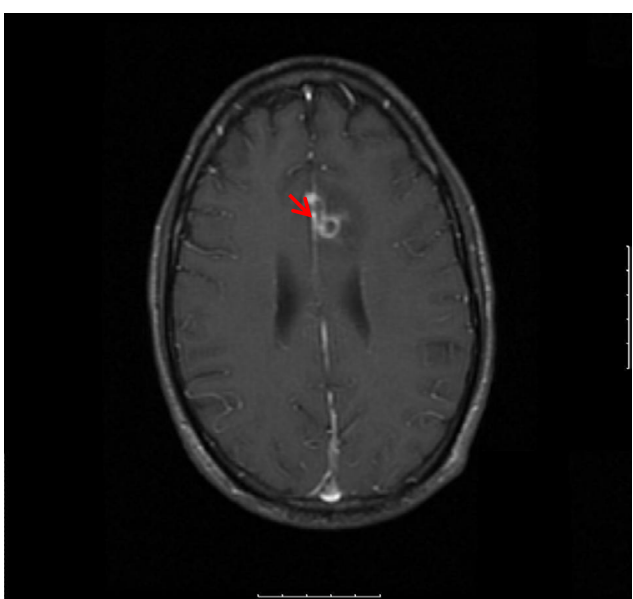

Figure 6: MRI scan of brain shows $1 \mathrm{~cm}$ brain abscess on the left

improved remarkably within the next few days. His repeat blood cultures were negative on day 4 and his symptoms resolved. Although it was advised that he continue the same antimicrobial regimen for an extended period of time, he was unwilling to do so. He was finally discharged on day 39 on a regimen of oral azithromycin, ethambutol and moxifloxacin. The patient was lost to follow up. He was readmitted a year later for prosthetic valve endocarditis due to coagulase negative staphylococcal species.

\section{Discussion}

Infective endocarditis due to mycobacterium species is an unusual but well defined clinical entity. Nontuberculous mycobacteria (NTM) have been reported more frequently as a cause of infective endocarditis than Mycobacterium tuberculosis, especially of prosthetic valves [1,2]. The previously identified causative NTM species have included the following rapidly growing mycobacteria: M. fortuitum, M. abscessus, and M. chelonae [2,3]. M. neoaurum, a member of the Mycobacterium parafortuitum complex, has not been reported previously to cause infective endocarditis.

Mycobacterium neoaurum has been reported rarely as a cause of bloodstream infections in immunocompromised hosts, such as patients with malignancies or transplant recipients [4]. M. neoaurum has also been implicated in pulmonary infections [5], meningoencephalitis [6], urinary tract infection [7] and catheter related infections [4,8].

NTM PVE has been described in both mechanical and biologic valvular prostheses. Although the origin of NTM infection of prosthetic valves is unclear, the most recent reports suggest a nosocomial source [9]. A recently proposed mechanism is the contamination of the prosthetic valve before or during the surgical procedure [3]. Many rapidly growing mycobacteria have been noted to contaminate water systems in hospitals: water supplies to operating rooms, sterilization fluids, cardioplegic and valve preservation solutions $[3,4]$. This is likely due to the organism's relative resistance to chemical decontamination. The indolent nature of these mycobacteria also allows for the delayed onset of symptoms and signs in cases of PVE in which perioperative contamination was suspected [3]. However, reports of M. neoaurum contaminating hospital water systems are not available at this time $[4,10]$. It is unclear how the prosthetic valve of our patient was infected with this organism. Mycobacterium neoaurum is found in the environment, and may likely have gained access to the bloodstream via breaks in the skin during intravenous drug use.

M. neoaurum can be detected on routine aerobic blood culturing systems and typically grows within 5 days on Lowenstein-Jensen agar. In many of the reported cases of endocarditis due to NTM the blood cultures were negative. The identification of the NTM was obtained by culturing the removed prosthetic valve, or by histopathologic analysis in conjunction with molecular assays. The advanced methods for identification include high-pressure liquid chromatography and sequencing of a unique $16 \mathrm{~S}$ ribosomal RNA [11]. Therefore, if blood cultures are negative in a patient suspected of having PVE, definitive diagnosis can be made by removal of the infected prosthetic valve and performance of the aforementioned studies. In an immunocompromised patient with unexplained fever, isolation of M. neoaurum, particularly in multiple specimens, should be considered highly suggestive of infection with this organism.

Susceptibility testing of rapidly growing mycobacteria can be clinically useful, although it is not standardized and results are often delayed well into empiric therapy. Presently, the minimum recommendations for antibiotic susceptibility testing of rapidly growing NTM include clarithromycin, amikacin, cefoxitin, imipenem (the carbapenem preferred over meropenem and ertapenem), tobramycin, doxycycline, linezolid, ciprofloxacin, and sulfonamides [12]. Ciprofloxacin resistance is now widely encountered; however, moxifloxacin should be tested as some NTM isolates remain susceptible to this fluoroquinolone. M. neoaurum has a better susceptibility profile compared with $M$. chelonae and $M$. abscessus. However, a recent study did demonstrate high Mycobacterium neoaurum resistance to clarithromycin [12]. Combination antimicrobial therapy including macrolides, fluoroquinolones and aminoglycosides is the preferred treatment regimen.

Successful treatment of $M$. neoaurum bloodstream infection most commonly requires several weeks of combination antimicrobial therapy and removal of prosthetic material. Despite these complex treatment regimens and removal of prosthetic material, the prognosis of the 
affected individuals with NTM PVE is poor. However, in contrast with patients who have PVE due to other NTM, our patient responded very well to antibiotic treatment. This could be due to the indolent nature and low pathogenicity of M. neoaurum. This is further corroborated by the fact that the majority of patients who had infections of indwelling catheters due to $M$. neoaurum responded very well to antibiotics and catheter removal.

In conclusion, NTM PVE is a rare clinical entity that should be considered in patients with prosthetic valves who present with symptoms of endocarditis with negative blood cultures. M. neoaurum is a rapidly growing NTM that can cause infective endocarditis in immunosuppressed individuals. Whether it can be nosocomially transmitted is yet to be proven. NTM PVE due to M. neoaurum, in contrast to PVE due to other NTM, may be amenable to long term antibiotic therapy, especially if combined with prosthesis removal. In the absence of definitive regimens and substantial experience, physicians can rely on antibiotic susceptibility data and previous case reports to guide their antibiotic choice and duration of therapy. In general, however, endocarditis secondary to NTM, particularly involving the prosthetic valves, carries a poor prognosis.

\section{References}

1. Corrales-Medina V, Concha R, Simkins J, Sanchez M, Baracco G (2007) Native valve endocarditis caused by rapidly growing mycobacteria: case report and review of the literature. Scand J Infect Dis 39: 639-641.

2. Olalla J, Pombo M, Aguado JM, Rodríguez E, Palenque E, et al. (2002) Mycobacterium fortuitum complex endocarditis-case report and literature review. Clin Microbiol Infect 8: 125-129.
3. Strabelli TM, Siciliano RF, Castelli JB, Demarchi LM, Leão SC, et al. (2010) Mycobacterium chelonae valve endocarditis resulting from contaminated biological prostheses. J Infect 60: 467-473.

4. Washer LL, Riddell J 4th, Rider J, Chenoweth CE (2007) Mycobacterium neoaurum bloodstream infection: report of 4 cases and review of the literature. Clin Infect Dis 45: e10-13.

5. Morimoto Y, Chan ED, Heifets L, Routes JM (2007) Pulmonary infection with Mycobacterium neoaurum identified by $16 \mathrm{~S}$ ribosomal DNA sequence. J Infect 54: e227-231.

6. Heckman GA, Hawkins C, Morris A, Burrows LL, Bergeron C (2004) Rapidly progressive dementia due to Mycobacterium neoaurum meningoencephalitis. Emerg Infect Dis 10: 924-927.

7. Zanetti S, Faedda R, Fadda G, Dupré I, Molicotti P, et al. (2001) Isolation and identification of Mycobacterium neoaurum from a patient with urinary infection. New Microbiol 24: 189-192.

8. Holland DJ, Chen SC, Chew WW, Gilbert GL (1994) Mycobacterium neoaurum infection of a Hickman catheter in an immunosuppressed patient. Clin Infect Dis 18: $1002-1003$

9. Bush LM, Paturi A, Chaparro-Rojas F, Perez MT (2010) Mycobacterial prosthetic valve endocarditis. Curr Infect Dis Rep 12: 257-265.

10. Covert TC, Rodgers MR, Reyes AL, Stelma GN Jr (1999) Occurrence of nontuberculous mycobacteria in environmental samples. Appl Environ Microbiol 65: 2492-2496.

11. Griffith DE, Aksamit T, Brown-Elliott BA, Catanzaro A, Daley C, et al. (2007) An official ATS/IDSA statement: diagnosis, treatment, and prevention of nontuberculous mycobacterial diseases. Am J Respir Crit Care Med 175: 367 416.

12. Brown-Elliott BA, Wallace RJ Jr, Petti CA, Mann LB, McGlasson M, et al. (2010) Mycobacterium neoaurum and Mycobacterium bacteremicum sp. nov. as causes of mycobacteremia. J Clin Microbiol 48: 4377-4385.
This article was originally published in a special issue, Pulmonology- Case Reportshandled by Editor(s). Dr. Anna Blumental-Perry, Memorial University Medical Center, USA. Dr. Bradley Allen Maron, Brigham and Women's Hospital, USA. 